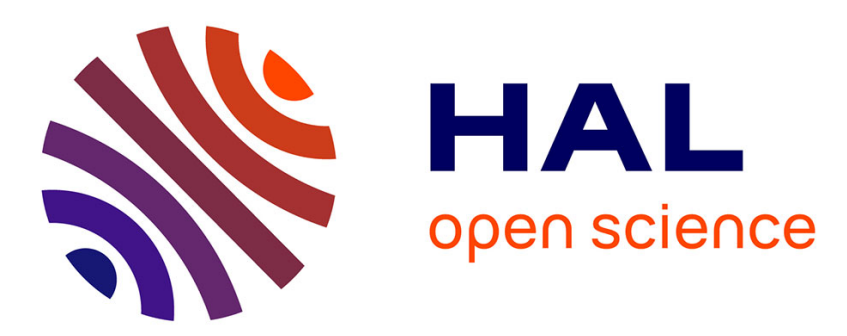

\title{
Vous avez le droit de garder le silence: un scénario pédagogique pour faire parler les étudiants de droit Sandrine Chapon
}

\section{To cite this version:}

Sandrine Chapon. Vous avez le droit de garder le silence: un scénario pédagogique pour faire parler les étudiants de droit. Recherche et Pratiques Pédagogiques en Langues de Spécialité: Cahiers de l'APLIUT, 2011, XXX (1), http://apliut.revues.org/477. hal-00911627

\section{HAL Id: hal-00911627 https://hal.science/hal-00911627}

Submitted on 29 Nov 2013

HAL is a multi-disciplinary open access archive for the deposit and dissemination of scientific research documents, whether they are published or not. The documents may come from teaching and research institutions in France or abroad, or from public or private research centers.
L'archive ouverte pluridisciplinaire HAL, est destinée au dépôt et à la diffusion de documents scientifiques de niveau recherche, publiés ou non, émanant des établissements d'enseignement et de recherche français ou étrangers, des laboratoires publics ou privés. 


\title{
Sandrine Chapon
}

\author{
Université Pierre Mendès-France Grenoble
}

\section{Vous avez le droit de garder le si- lence : un scénario pédagogique pour faire parler les étudiants de droit}

\section{You have the right to remain silent: A learning scenario to enhance oral communication in Legal English classes}

Key words: Legal English, self-learning environment, institutional goal, methodological goal, TV series as resource material. tif méthodologique, séries télévisées comme support pédagogique.

Résumé : Le volume horaire des langues à la Faculté de Droit de Grenoble a diminué de moitié en 5 ans. Il est donc nécessaire d'adapter la pédagogie de projet afin de concilier les besoins des apprenants et la diminution des moyens à leur disposition. Le scénario pédagogique que nous proposons de présenter s'articule autour du légendaire cinquième amendement de la Constitution américaine. Il tente de respecter la démarche actionnelle dans laquelle tous les étudiants peuvent prendre une part active à leur apprentissage dans un contexte peu propice à la production orale. Les phases de production font l'objet d'un travail en autonomie guidée. La tâche finale de prise de parole en continu est rendue possible en utilisant des moyens multimédia : lecteurs MP3 ou téléphones portables des étudiants comme ressources documentaires et moyens d'enregistrement.
Abstract: In the past five years, the number of hours dedicated to learning foreign languages at the Grenoble Law School has been reduced by half making it necessary to design teaching strategies to match the drastic decrease of means while meeting the learners' needs. The following learning scenario is based on the fifth amendment of the American Constitution. It focuses on task-based language learning where every student can take an active part in the learning process in an environment unfavourable to the practise of oral skills. The task involving oral production is carried out outside the classroom and sent via e-mail to the teacher for assessment. The students use their mobile phones or MP3 players in order to record their production. 
Sandrine Chapon*

Université Pierre Mendès-France Grenoble

\section{«Vous avez le droit de garder le silence » : \\ Un scénario pédagogique pour faire parler les étudiants de droit.}

Semblable à la partie immergée de l'iceberg, la phase de conceptualisation des dispositifs didactiques représente, en temps de mise en œuvre, l'aspect le plus prenant de la fonction d'enseignant. En effet, quand il conçoit des projets pédagogiques dans le secteur LANDSAD (LANgues pour Spécialistes d'Autres Disciplines), l'enseignant doit prendre la mesure d'un certain nombre de variables: le niveau langagier de départ du public visé, les compétences à atteindre, la spécificité de la langue culture de spécialité, le volume horaire et le format des cours définis par le « règlement d'études ${ }^{1} »$ des étudiants. Toute la problématique réside dans la capacité à intégrer chacun des paramètres sans sacrifier l'objectif d'insertion professionnelle sur l'autel de la rigueur budgétaire. Certains postes demandant une excellente maîtrise de l'anglais, comment répondre à cette exigence malgré des contraintes externes fortes (peu d'heures de cours en première année, dispensées pour moitié en amphi)?

Cet article a pour vocation la présentation synoptique d'un scénario pédagogique visant à placer les compétences de communication au cœur de la situation d'apprentissage, malgré un format de cours peu propice à cet objectif.

Après avoir présenté le cadre institutionnel dans lequel s'inscrivent les cours de langues à la Faculté de Droit de Grenoble, je mettrai en perspective le niveau des primo-entrants et les attentes professionnelles.

Le scénario s'intitule « Vous avez le droit de garder le silence », en référence à la procédure policière américaine puisqu'il prend son ancrage culturel dans l'application du célèbre cinquième amendement de la Constitution américaine par le biais des séries télévisées. L'unité didactique présentée sera une des facettes d'un projet de création d'un site d'auto-formation à la langue étrangère alimenté par les étudiants eux-mêmes.

\footnotetext{
* Sandrine Chapon, professeure certifiée, enseigne l'anglais juridique à la Faculté de Droit de Grenoble depuis 5 ans. Elle est chargée de mission aux langues et coordinatrice de l'équipe d'enseignants d'anglais pour la mise en œuvre d'un projet pédagogique visant à adapter la démarche actionnelle au nouveau format de cours pour les L1 (alternance de cours en amphi et TD), applicable à la rentrée 2011. <sandrine.chapon@upmf-grenoble.fr>.

${ }^{1}$ Nom officiel du programme à la faculté de droit de Grenoble.
} 
La dernière partie servira à montrer que le dispositif comporte aussi une dimension méthodologique de prise de conscience des moyens informels d'apprentissage de la langue et d'incitation au travail en autonomie.

\section{Diagnostic}

Jusqu'en 2005, l'enseignement des langues en licence était une option à choisir parmi une offre extrêmement vaste. Le rythme de cours était fixé à 1,5 heures hebdomadaires, soit un total de 36 heures par an. En 2007, l'enseignement des langues est devenu obligatoire en L3 avec, pour corollaire, une diminution progressive à $24 \mathrm{~h}$, ce qui a rendu possible le regroupement de tous les cours sur un seul semestre à raison de 2 heures par semaine. Désormais, les étudiants peuvent rester jusqu'à 8 mois sans pratiquer de langue étrangère. Si l'on compare la situation avec la formation «Carrière juridique » de l'IUT, les étudiants de la Faculté de Droit ont 2/3 d'heures de moins ( $24 \mathrm{~h}$ contre $76 \mathrm{~h}$ ).

Conscient de la faiblesse de l'appareillage éducatif, le Doyen, sous l'impulsion de la politique des langues de l'Université, a pris la décision de rendre obligatoires les langues pour toutes les années à partir de la rentrée 2011, ce qui correspond à la nouvelle maquette d'habilitation ministérielle.

Afin de résoudre le problème du très grand nombre d'inscrits en première année (quelque 700 étudiants), le format retenu pour les cours de L1 est une alternance de cours dispensés en amphi et en TD.

Au regard de l'évolution de ces cinq dernières années, la décision de supprimer le caractère facultatif de l'enseignement des langues vient remettre une certaine cohérence dans le parcours éducatif des étudiants. En effet, l'anglais est une épreuve orale obligatoire dans un grand nombre de concours accessibles avec un bac +4 ou + 5 (concours d'entrée à l'École Nationale de la Magistrature, Commissaire de Police, Directeur de Prison, École des Commissaires aux Armées, par exemple).

Cette modification est, en ce sens, une avancée positive car, en plus de ces concours, la Faculté de Droit a vocation à préparer à des carrières professionnelles qui exigent une très bonne maîtrise de la langue anglaise, notamment les carrières juridiques internationales, les domaines d'activités relatives aux droits de l'homme, à la propriété intellectuelle, aux métiers des projets internationaux de coopération, aux juristes-conseils d'affaires ou aux postes dans les instituts de défense et de sécurité internationale (Ministère de la défense, Collège de défense de l'OTAN, Institut d'études de sécurité de l'Union Européenne, etc.). Cette finalité professionnelle est, par exemple, mentionnée dans le descriptif du Master 2 Droit des entreprises, juristes-conseils d'affaires :

La dimension internationale et communautaire est consubstantielle à la matière du droit des affaires : les futurs diplômés du Master spécialisé Droit des entreprises, Juristes-conseils d'affaires ont donc tout naturellement vocation à travailler soit à l'étranger, soit dans des entreprises ou des organismes à dimension internationale. 
$-120-$

La problématique qui nous anime est donc d'atteindre l'objectif professionnel en termes de compétences linguistiques à partir des données initiales. Cette mission peut paraître ambitieuse si l'on se fonde sur les résultats d'un test de positionnement réalisé par l'Université Pierre Mendès-France (secteur des sciences sociales et humaines) à l'automne 2009 et qui révèle que $90.7 \%$ des étudiants qui entrent en L1 ont un niveau inférieur à B22 .

Ces chiffres rendent nécessaires de s'interroger sur la stratégie d'apprentissage et excluent de proposer des enseignements de type EMILE (Enseignement d'une Matière Intégré à une Langue Étrangère) pour la partie des cours en amphi, comme envisagé par l'administration. Nous avons préféré conceptualiser des dispositifs pédagogiques qui utilisent toutes les compétences linguistiques, malgré le format des cours peu propice à la prise de parole. L'appareil pédagogique que nous allons présenter permet par ailleurs de développer des modules de travail en autonomie afin d'encourager les étudiants à pratiquer la langue pendant la très longue période sans cours.

\section{Mise en œuvre}

\subsection{Ancrage culturel}

Le fil conducteur du scénario pédagogique est l'arrêt Miranda vs Arizona, très largement représenté dans les films et téléfilms américains dans les scènes où un officier de police fait lecture de ses droits à un suspect lors de son arrestation. Le recours à l'avertissement « Vous avez le droit de garder le silence $[\ldots]^{3}$ » est communément nommé Miranda rights / Miranda warning par les locuteurs natifs américains.

L'origine de l'expression prend ses sources dans une affaire au pénal dans laquelle le défendeur Ernesto Miranda, violeur récidiviste a été mis en examen sur le fondement de ses aveux lors d'un interrogatoire de police en 1966. Il a été relaxé devant la juridiction de jugement parce que la police ne lui avait pas signifié ses droits avant de le questionner. L'affaire a été portée devant la Cour Suprême qui a statué sur l'admissibilité des aveux lors de l'enquête en faveur du défendeur.

La décision est fondée sur deux amendements du Bill of Rights : le cinquième amendement prévoit que nul ne peut être forcé à témoigner contre lui-même : «No person [...] shall be compelled in any criminal case to be a witness against himself $\gg)$.

Le sixième amendement dispose que l'accusé a droit à un avocat : "In all criminal prosecutions, the accused shall enjoy the right [...] to have the Assistance of Coun-

\footnotetext{
${ }^{2}$ Chiffres communiqués par Séverine Wozniak, Université Grenoble 2, coordinatrice du dispositif de positionnement-remédiation des L1 Économie-Gestion (UFR d'Économie).

3 "You have the right to remain silent."
} 
sel for his defence. »).

Le patronyme Miranda a subi une évolution tropologique (Isani 2005 : 91) puisqu'il est depuis utilisé comme un nom commun : "If it's an arrest that's clearly imminent, Miranda must be read! $!^{4} \gg$ ou un verbe, to Mirandize. Il se rapporte donc au respect des droits fondamentaux des citoyens américains.

\subsection{Choix d'outils pédagogiques}

Les supports sélectionnés pour le cours sont, entre autre, des FASP (Fiction à Substrat Professionnel) télévisuelles juridiques et policières. Selon la définition de Petit (2004 : 188), ce sont des thrillers qui mettent en scène des professionnels (des avocats et des policiers) dans le contexte culturel étudié. Chacun des extraits proposés comporte une occurrence du trope éponyme Miranda.

En plus des FASP, un extrait d'une série non FASP, Desperate Housewives, a été sélectionné dans le but de montrer que ce trope n'appartient pas exclusivement à la communauté linguistique des hommes de loi (avocats, magistrats) ou de la police, comme pour les autres extraits, mais qu'il bénéficie, grâce à la culture télévisuelle des Américains, d'une reconnaissance mutuelle par des locuteurs non spécialistes de l'anglais juridique (Isani 2005 : 97).

\subsection{Mise en train}

Le dispositif didactique a été testé dans un premier temps sur deux groupes de L2 en 2010. Quatre heures sont nécessaires pour mener à bien les séquences présentées dans cet article.

La première tâche demandée aux étudiants est la traduction, avec l'aide du dictionnaire, de deux citations tirées d'une FASP juridique, The practice ${ }^{5}$, mettant en scène les associés d'un cabinet d'avocats qui discutent de leur client :

- «They haven't given him Miranda».

- «Did the man get Miranda?»

Ce stimulus vise à déclencher deux types d'actions : une exécution de la tâche doublée d'une réflexion métacognitive sur le sens de cette tâche, car le premier réflexe des étudiants est d'exprimer la difficulté à observer la consigne en l'absence de contexte. En effet, la propriété particulièrement polysémique du verbe to get (22 entrées dans le dictionnaire Robert \& Collins) rend la tâche hasardeuse. Par contre, le seul questionnement qui n'est pas opéré par les étudiants est la remise en question de l'identité sexuelle de Miranda car le nom propre est en apparence univoque et est automatiquement identifié comme un prénom féminin.

On obtient des propositions de traductions radicalement différentes : «Est-ce que

\footnotetext{
${ }^{4}$ Shark, saison 1, épisode 10.

${ }^{5}$ Série américaine écrite par David E. Kelley et diffusée sur le réseau ABC entre 1997 et 2004.
} 
l'homme a réussi à joindre Miranda, à l'appréhender, à la trouver, à aller la chercher, à la comprendre », etc.

La traduction la plus couramment proposée pour «they haven't given him Miran$d a »$ est «ils ne lui ont pas remis Miranda », les étudiants émettant l'hypothèse que Miranda est un COD non humain et serait plutôt un chien, un dossier secret du FBI, un nom de code de la CIA, etc. Aucun d'entre eux ne suggère, par contre, qu'il puisse faire référence à la fille de Prospero dans The Tempest de Shakespeare.

\subsection{Liaison culture-lexique}

\section{- Travail d'inférence}

Cette activité va consister à valider / invalider les hypothèses émises en amont par les étudiants en visionnant plusieurs extraits de séries américaines afin de les entraîner aux opérations mentales de déduction grâce au contexte. Hutchinson et Waters (1987 : 140) expriment en termes motivationnels la démarche qui sous-tend cette stratégie :

Quand les gens font des prédictions, ils placent une partie de leur amour-propre dans les décisions et les choix qu'ils opèrent. Ils mettent en jeu leur ego et prennent des risques. Ils seront alors obnubilés par une chose : découvrir s'ils avaient raison ${ }^{6}$. (Notre traduction).

Chaque extrait comporte l'utilisation d'une forme du trope qu'il conviendra de repérer dans un objectif de discrimination orale :

\footnotetext{
«We don't really believe in Miranda anymore ». (Boston Legal, saison 2, épisode 10)

«Did the man get Miranda? » (The Practice, saison 1, épisode 04)

«They haven't given him Miranda or anything? (The Practice, saison 1, épisode 08)

«Let's make clear that Mr Bratton has not been mirandized and that what is said here is for the purpose of a proffer. Agreed? ». (The Wire, saison 1, épisode 11)

"If it's an arrest that's clearly imminent as it was here, Miranda must be read! ». (Shark, saison 1, épisode 10)

"You're a suspect. They have the right to question you. Just listen to me. Tell them you will not waive your Miranda rights and we'll have a lawyer down there as soon as we can. Remember: how does a fish get caught? He opens his mouth ». (Desperate Housewives, saison 6, épisode 02)
}

Les éléments qui permettent d'inférer la signification du verbe to Mirandize sont récurrents :

- les personnages présents dans les scènes ou auxquels on se réfère : police officers, lawyers, suspects ;

- les lieux : crime scene, interrogation room;

\footnotetext{
6 "When people make predictions, they are investing part of their self-esteem in their decisions and choices. They are putting their ego on the line and taking risks. Having done so, the one thing that will occupy their minds is finding out whether they were right."
} 
- des verbes : to arrest, to talk, to question, to open one's mouth;

- des substantifs : rights, an arrest, a suspect.

La convergence des informations rend possible l'opération de déduction de la forme to Mirandize sb vers le sens to tell sb his/her rights.

\title{
- Activité de transformation
}

Une fois le travail de décodage du trope effectué, on peut proposer aux étudiants de reconsidérer leurs traductions initiales et de suggérer des productions plus en adéquation contextuellement avec les scènes observées.

Pour les citations : «They haven't given him Miranda » et «Did the man get Miranda? », on obtient des propositions du type «On lui a signifié / lu / dit ses droits ».

Dans l'objectif de valoriser la nouvelle posture d'expert de la langue de spécialité des étudiants, nous proposons de repasser un des extraits visionnés précédemment avec, cette fois, les sous-titres en français afin que les étudiants comparent leur traduction avec celle du DVD. Quand ils lisent que le sous-titrage de la bande-son «Did the man get Miranda? » est «A-t-il appelé Miranda?», le sentiment de «complicité et de connivence interactives » né de l'intercompréhension du trope (Isani 2005 : 97) est palpable ! Le ressenti agréable vient de ce que, à l'évidence, le traducteur officiel de l'épisode ne possède pas les clés du «code restreint spécifique à la communauté » identifié par Isani alors que les étudiants sont désormais capables de décrypter ce nom propre tropologique. Cet exercice les place dans une posture flatteuse et, par là-même, propice à l'activité cognitive.

Tout en exerçant l'oreille à la discrimination orale, cette première phase du scénario est fondée sur l'expérience agréable de revisiter des séries américaines que les étudiants regardent par ailleurs, et sur la découverte du phénomène du trope qui suscite la stimulation intellectuelle :

\begin{abstract}
Il y a donc un défi que l'on peut qualifier de ludique et qui consiste à décoder le ou les messages qui auraient été insérés ou «encodés » selon l'expression de Stuart Hall, dans le texte télévisuel. La satisfaction procurée par ce décryptage provient de la conscience que l'on a de participer à la construction du sens du récit et de se sentir plus malin, attribut cher à la culture française (Villez 2005 : 127).
\end{abstract}

\subsection{Compétences linguistiques}

\section{- Activités de réception}

La séquence suivante vise à mettre en œuvre des capacités de communication dans le contexte posé initialement. La première tâche proposée sert à comprendre l'origine du trope Miranda grâce à un texte puisé sur le site Wikipedia concernant Ernesto Miranda. Le document permet d'appréhender le contexte de la décision de l'arrêt Miranda vs Arizona qui a fait jurisprudence. Cette activité de scanning a pour but le repérage des informations essentielles qui seront utilisées ultérieurement. 
La deuxième tâche a pour objectif culturel d'observer le caractère itératif du phénomène linguistique de néologie par conversion dans la langue anglaise (Google $\rightarrow$ to google). Par ailleurs, l'activité permet de développer la compétence de compréhension orale à travers l'étude de noms déposés tropologiques. Sur le site $B B C$ learning English section «for teachers», à la rubrique «keep your English up to date », David Crystal présente des expressions idiomatiques repérées dans l'anglais contemporain. Ces discours oraux, téléchargeables au format MP3, décrivent l'origine étymologique, l'époque d'intégration dans la langue et l'évolution morphosyntaxique de ces syntagmes lexicalisés.

Parmi les termes proposés à l'étude sur le site, Google et Facebook ont été sélectionnés en fondant notre choix sur l'importance de prendre en considération les intérêts des apprenants. Facebook est le deuxième site web le plus utilisé au monde après Google et $95 \%$ des étudiants de la Faculté de Droit interrogés y ont un compte. Ces tropes sont donc en concordance avec leurs habitudes de réseautage social et il est amusant pour eux de découvrir le haut degré d'adaptabilité de la langue anglaise et la manière dont les locuteurs natifs s'emparent notamment des noms déposés pour en faire des outils sémantiques comme en attestent les exemples proposés par David Crystal : "I facebooked Mary about the party», «he's google-minded», etc.

Après avoir proposé des activités de compréhension globale puis détaillée de ces deux discours, nous proposons d'utiliser ces ressources comme palimpseste.

\section{- Prise de parole en continu}

L'étudiant est invité à effectuer un exercice d'écriture parallèle en adaptant le schéma formel proposé par le document authentique et en le transformant pour réaliser sa propre création. La tâche consiste à travailler en imitation de David Crystal, en rédigeant puis en oralisant un discours métalinguistique sur le trope Miranda d'une durée de 3 minutes maximum. Les étudiants doivent ensuite s'enregistrer sur leur téléphone portable ou leur ordinateur personnel et envoyer leur fichier par courriel à l'enseignant qui pourra procéder à une évaluation formatrice des productions. Les critères retenus sont d'ordre prosodique (rythme, intonation) et permettent également de mesurer l'écart entre le code choisi et l'intention de communication. Des stratégies de remédiation pourront être mises en œuvre lors des cours de TD, pour aider les étudiants à surmonter leurs difficultés.

\section{- Tâche finale}

La tâche finale, une méso tâche de prise de parole en continu, s'inscrit dans le projet de réaliser à moyen terme un site d'espace collaboratif d'auto-apprentissage de l'anglais juridique alimentée, entre autres, par les étudiants. Quelques-unes des productions les plus abouties en termes de réalisation phonique pourront être mises en ligne. On pourra proposer à d'autres groupes l'étude de nouveaux lexèmes sur le même modèle. Cette série de productions viendra marquer la première pierre du projet qui a donc un objectif immédiat de travail des compétences linguistiques et 
une visée à plus long terme de construction d'un savoir interculturel à partager avec les autres apprenants. Le scénario pédagogique qui utilise des fictions américaines a aussi pour ambition de servir de miroir aux étudiants afin de les encourager à développer leurs stratégies d'apprentissage informelle de l'anglais en dehors du cadre institutionnel.

\section{Compétence méthodologique}

Une étude menée en 2009 à la Faculté de Droit auprès de 55 étudiants de troisième année, dans le but de découvrir leur culture des séries américaines (Chapon 2010 : à paraître), révèle qu'ils connaissent en moyenne 18 séries mais que $65 \%$ d'entre eux les regardent en français. Par ailleurs, une enquête de la Commission Européenne (2006:48) montre que seuls $31 \%$ des Français interrogés sont d'accord avec l'affirmation «Je préfère regarder les films et programmes étrangers avec des soustitres plutôt que des doublages » (2006: 58). De surcroît, les Européens interrogés estiment à $57 \%$ que le meilleur endroit pour apprendre les langues étrangères reste la classe (2006 : 48). Ces enquêtes révèlent une grande disparité entre la perception subjective de la manière la plus efficace d'apprendre une langue étrangère et certaines données statistiques exposées dans l'article de Micola et al. (2009 : 15).

Ces auteurs analysent l'impact de la politique culturelle des pays sur la maitrise de l'anglais suivant qu'ils choisissent le doublage des voix ou le sous-titrage de la version originale des programmes télévisés étrangers. Leur étude porte sur 32 pays dont l'anglais est la langue étrangère la plus utilisée. Elle montre que, dans les états qui ont opté pour le sous-titrage, les résultats sont bien meilleurs, surtout dans les compétences de compréhension orale (près de deux fois plus) et obtiennent en moyenne 77 points de plus au TOEFL que dans les pays qui choisissent de doubler leurs programmes.

L'observation empirique de Laure Peskine ${ }^{7}$, «Je le vois bien en cours, les élèves qui regardent des films en version originale ont une meilleure compréhension orale. Ils sont habitués à la musique de la langue », nous est familière. Dans la perspective didactique qui est la nôtre, nous pouvons intégrer ces données au projet pédagogique pour mettre en œuvre une stratégie de valorisation des pratiques informelles des étudiants dans le but de faire véritablement prendre conscience du potentiel des fictions américaines, regardées en version originale, pour l'amélioration des compétences langagières.

Selon Bonnet (2002: 107), les enseignants français encouragent moins souvent leurs élèves à pratiquer la langue en dehors de la classe que leurs homologues européens. Didactiser la fiction peut donc servir à valider la pratique des étudiants (Toffoli et al. 2010 : 137). Cette validation est nécessaire puisque l'étude de la Commission Européenne (2006 : 48) montre que seuls $12 \%$ des Européens inter-

\footnotetext{
${ }^{7}$ Secrétaire nationale de l'Association des Professeurs de Langues Vivantes, interrogée pour le magazine de la FCPE La revue des parents, juin 2010.
} 
$-126-$

rogés pensent que regarder les films en version originale est une manière efficace d'apprendre les langues. Il appartient donc à l'enseignant de porter à la connaissance des étudiants cette stratégie d'apprentissage pour la rendre consciente.

Toffoli et al. (2010 : 138) montrent que l'une des applications pédagogiques possibles de l'apprentissage informel de la langue cible en dehors de la classe peut être d'inverser les rôles apprenants/enseignant en demandant que les étudiants soient, pour un temps donné, les fournisseurs des supports pédagogiques. En les invitant par exemple à venir présenter un passage d'une des séries qu'ils affectionnent où leur héros rencontrerait un problème lié à la loi, pour ce qui nous concerne. On peut aussi imaginer qu'ils soient incités à trouver une scène montrant une particularité culturelle juridique, le phénomène de la maternité de substitution (surrogacy) aux États-Unis ou des peines alternatives infamantes (shaming sentences), par exemple. L'enseignant encouragerait à regarder la série comme «miroir de la société » (Boutet 2009 : 56). À l'issu du scénario sur le trope Miranda, des étudiants ont exprimé leur étonnement concernant la quantité d'extraits de films mentionnant ce lexème. Nous leur avons donc expliqué que nous «collectionnions » ce trope à des fins de traduction comparée et les avons conviés à se joindre à notre recherche. Nous avons de temps en temps l'agréable surprise de recevoir un courriel d'un étudiant disant qu'il a entendu l'expression dans un épisode et donnant les références de la série en question.

\section{Conclusion}

Du point de vue de la motivation, les supports télévisuels sont plébiscités par les apprenants qui y trouvent un intérêt grâce à la proximité affective avec les personnages. Le public visé étant essentiellement un panel d' «utilisateurs élémentaires » de la langue anglaise selon le descripteur du Cadre Européen Commun de Référence pour les Langues du Conseil de l'Europe, la vidéo est aussi un moyen d'accéder au sens grâce aux informations portées par l'image.

Les objectifs qui ont été décrits ici de manière asynchrone par souci de clarté, sont nécessairement interdépendants. Tout en cherchant à sensibiliser les étudiants à l'altérité des cultures, le scénario propose une réflexion sur la langue dans un dispositif qui permet aux étudiants de se rendre compte que la langue étrangère offre «une représentation du monde non superposable culturellement» (Quivy 2005 : 272) à leur langue maternelle.

L'unité didactique propose aussi une manipulation du dictionnaire dans le but de favoriser l'appropriation de la capacité transversale d'utilisation de l'outil de référence. Elle vise également la démarche cognitive qui comprend des «objectifs intellectuels, la réflexion grammaticale directement utile à la formation intellectuelle, les capacités de raisonnement et d'abstraction » (Puren 1999 : 177).

Par ailleurs, les étudiants sont invités à développer des attitudes de coopération en participant collectivement à la construction du sens interculturel, en se plaçant dans 
la posture d'acteurs sociaux qui vont réaliser une tâche, celle de la construction du contenu d'un site d'apprentissage pour leurs pairs.

Toffoli et al., citant Cross (2010:126), définissent l'apprentissage informel comme la manière «non officielle, non planifiée et impromptue » d'acquérir une expertise professionnelle. Cet apprentissage involontaire est aussi observable dans l'action de regarder pour le plaisir des programmes en langue étrangère en version originale sous-titrée (Micola et al. 2009). Dans la mission de l'enseignant figure ce que les Instructions officielles de 1989 appellent «apprendre à apprendre ». Dans une perspective de clarification des stratégies, il est donc intéressant d'inscrire cet apprentissage informel au cœur du projet pédagogique en didactisant des séries (Chapon: 2010). La FASP fournit justement des documents authentiques de grande valeur qui peuvent être étudiés comme le produit d'une culture sociétale et professionnelle (Isani : 2010).

\section{Références bibliographiques}

BONNET, Gérard (éd.) 2002. "The assessment of pupils' skills in eight European countries. A European project ».

$<$ http://www.institutodeevaluacion.educacion.es/contenidos/internacional/Assessm ent_of_English_BED.pdf>. Consult. 16 mai 2010.

BOUTET, Marjolaine. 2009. Les séries télévisées pour les nuls, Paris : First Editions.

CHAPON, Sandrine. Juin 2010. «FASP juridique télévisuelle et cinématographique : Gros plan sur les étudiants de droit et zoom arrière sur une pratique pédagogique ». Communication lors des journées d'étude «La FASP, une autre voie d'accès aux langues de spécialité : enjeux didactiques ». 9 et 10 juin 2010. Université de Caen Basse-Normandie.

CONSEIL DE L'EUROPE. Un cadre européen de références pour les langues : apprendre, enseigner, évaluer.

<http://www.coe.int/T/DG4/Linguistic/Source/Framework_FR.pdf>. Consult. 25 mai 2010.

EUROPEAN COMMISSION. 2006. Europeans and their languages. Special barometer $243<$ http://ec.europa.eu/education/languages/pdf/doc631_en.pdf>. Consult. 18 juin 2010.

HutChInSON Tom \& Alan WATERS. 1987. English for Specific Purposes, a learning-centred approach. Cambridge : University Press.

ISANI, Shaeda. Juin 2010. «Developing professional cultural competence: the multi-layered cultural substrata of the FASP ». Communication lors des journées d'étude «La FASP, une autre voie d'accès aux langues de spécialité : enjeux didactiques ». 9 et 10 juin 2010. Université de Caen Basse-Normandie.

ISANI, Shaeda. 2005. « Tropologie et noms propres en langues et cultures de spé- 
$-128-$

cialité : quelques considérations didactiques ». In FRIES Marie-Hélène (dir.). 2005. Métaphore et anglais de spécialité. Bordeaux : Travaux 20.25, 91-102.

Micola, Augusto, Arturo BRIS \& Albert BANAL-Estanol. «TV or not TV? Subtitling and English skills ». RePEc.

<http://www.econ.upf.edu/docs/papers/downloads/1156.pdf>. Consult. 16 mai 2010.

PETIT, Michel. 2004. «La Fiction à substrat professionnel : une voie d'accès à l'anglais de spécialité ». In IsANI Shaeda \& Michel PETIT (dir.). 2004. Aspects de la fiction à substrat professionnel. Bordeaux : Travaux 20.25, 187-208.

PEYRI, Marianne. 2010. «Inciter à voir les films en version originale». La revue des parents, vol. $\mathrm{n}^{\circ} 370: 11$.

PUREN, Christian. 1999. Se former en didactique de l'anglais. Paris : Ellipses.

QUIVY, Mireille \& Claire TARDIEU. 2005. [2002] Glossaire de didactique de l'anglais. Paris : Ellipses.

SOCKETT, Geoffrey. «Les processus cognitifs de résolution de problèmes pour l'apprentissage des langues dans des environnements multimédia ». In Actes du Colloque ACEDLE 2009.

<http://evenements.univ-lille3.fr/colloque-acedle2009/pdf/actes-colloque.pdf >

Consult. 16 mai 2010.

TOFFOLI, Denyse \& Geoffrey SocKETT. 2010. «How non-specialist students of English practice informal learning using 2.0 tools ». Asp, $\mathrm{N}^{\circ} 58: 125-144$.

VILLEZ, Barbara. 2005. Séries télé, visions de la justice. Paris : PUF.

\section{Références en ligne}

BBC Learning English.

$<$ http://www.bbc.co.uk/worldservice/learningenglish/teachingenglish/plans/uptodat e.shtml>. Consult. 03 juill. 2010.

Faculté de Droit de Grenoble: Master 2 Droit de l'entreprise, juristes-conseils d'affaires <http://webu2.upmf-grenoble.fr/facdroit/>, puis Formations / Diplômes de M1 et M2 / Master 2 Droit de l'entreprise, juristes-conseils d'affaires. Consult. 15 avr. 2010.

FindLaw for legal professionals.

$<$ http://caselaw.lp.findlaw.com/scripts/getcase.pl?navby=CASE\&court=US\&vol=3 84\&page $=436>$. Consult. 03 juill 2010.

'Ernesto Miranda' sur Wikipedia.

<http://en.wikipedia.org/wiki/Ernesto_Miranda〉. Consult. 15 déc. 2009. 